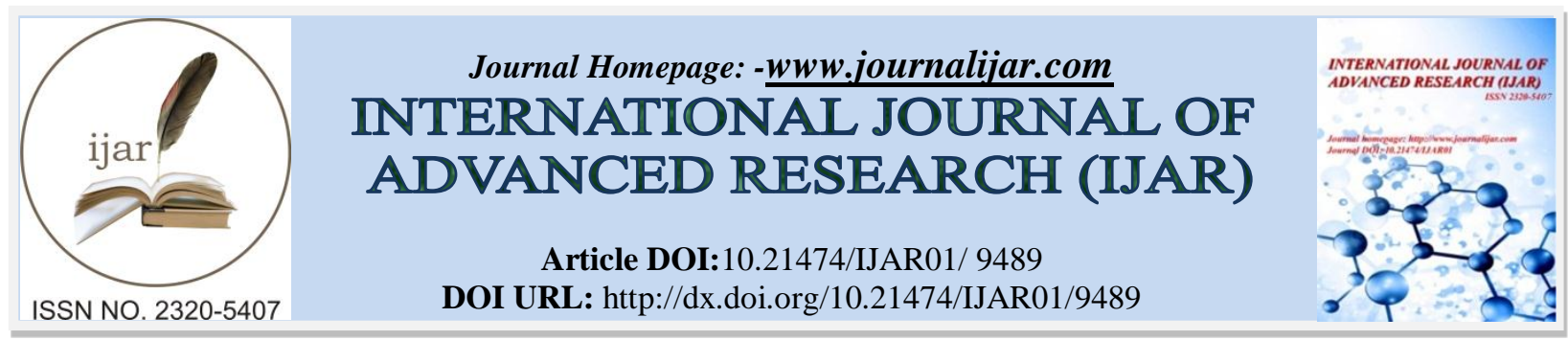

RESEARCH ARTICLE

\title{
CADCAM V/S CONVENTIONAL TECHNIQUE FOR BETTER MARGINAL FIT OF METAL COPINGS FROM WAX PATTERNS.
}

\section{Phani Chnadrika ${ }^{1}$,Chandrasekhar $N^{2}$, Soujanya $\mathrm{K}^{3}$, Ravalika $\mathrm{KN}^{3}$, Siddesh Kumar ${ }^{4}$ and Jagadeesh Konchada ${ }^{5}$.}

1. Private practitioner, Andhra pradesh, India.

2. Associate Fellow AAID, USA; Private practitioner, Dr. Sekhar's Dental Care and Implant Centre, Telangana, India.

3. Private practitioner, Dr. Sekhar's Dental Care and Implant Centre, Telangana, India.

4. Professor, Department of Prosthodontics, Sri Sai Dental College and Research Institute, Srikakulam, Telangana, India.

5. Reader, Department of Prosthodontics, Sri Sai Dental College and Research Institute, Srikakulam, Telangana, India.

\section{Manuscript Info}

\section{Manuscript History}

Received: 05 June 2019

Final Accepted: 07 July 2019

Published: August 2019

Key words:-

CAD/CAM, marginal fit, wax patterns.

\section{Abstract}

Aim: To evaluate and compare the marginal gap of metal copings cast from wax patterns fabricated with conventional technique and CAD/CAM technique.

Materials and Method: Fifty samples were fabricated using Standardized MIS implant abutment as a die. 25 wax patterns $(n=25)$ were fabricated by conventional wax coping method and rest $(n=25)$ were fabricated by CAD/CAM wax milling technique. The wax copings of both the methods are casted with conventional casting technique. The marginal gap of copings was evaluated by direct view technique under stereomicroscope (MAGNUS) with digital camera. Then images were transferred to personal computer for measuring marginal gap at 15 random points by using image analyzer (IMAGE PRO EXPRESS 6.0). Statistical data was calculated and further analyzed by unpaired student t-test $(\alpha=0.05)$.

Results: This study showed an overall mean of $595.26 \mu \mathrm{m}$ for absolute marginal discrepancy (AMD) with Standard deviation of 264.94 for CAD/CAM group and 233.42 um with Standard deviation of 184.98 for conventional group (control). Unpaired T-Test revealed that marginal gap was significantly higher in CAD CAM group than the conventional wax-up group.

Conclusions: Within limitations of this study conventional method of wax pattern fabrication produced copings with significantly better marginal fit than CAD/CAM technique. All the factors for 2 groups were standardized except wax pattern fabrication technique therefore only the conventional group results in copings with clinically acceptable margins of less than 300 microns. Advancement in digital dentistry has improved the ease and quality of restoration in dental lab but still needs a technical improvement in scanning procedure, data processing and milling technology. 
Clinical Significance: The long-term success rate of metal ceramic crown is determined by the proper marginal fit between the restoration and the prepared tooth. The improper fit or marginal discrepancy leads to secondary caries which accounts for $85 \%$ failures in fixed partial dentures. Even though digital technology has radically emerged into the dental laboratory procedures to improve the quality of restorations, this study reveals conventional wax up and casting procedure showed better marginal fit as compared to CAD-CAM system.

Copy Right, IJAR, 2019,. All rights reserved.

\section{Introduction:-}

Wax patterns are fabricated with wax using particular waxing instruments in conventional techniques. Rationale for choosing wax is that, it can be manipulated and shaped according to preferences and can also be completely eliminated from the mold by heating. Among the various steps of making the porcelain fused-metal crown, fabrication of the wax pattern is the most analytical, effortful and time-consuming step. Also, the wax-up's quality is dependent on the skill of the individual doing it. Previous research states that removing a wax pattern from a die with a shoulder margin causes an average of $35 \mu \mathrm{m}$ opening of the margin before investing. Wax has various integral restrains such as, fineness, thermal sensitivity, elastic memory and a high coefficient of thermal expansion. Also, the wax pattern's colour and glossy surface makes it difficult to identify minute defects. ${ }^{1}$

CAD/CAM technology has become a major revolution in modern dentistry and represents the most innovative approach. It is considered as an advancement over conventional dentistry as it allows extremely precise designs and good quality restorations. ${ }^{2}$ Today, due to the availability of various CAD/CAM systems, it is possible to fabricate the wax patterns made from castable materials, avoiding above stated limitations of wax and conventional wax-up technique. ${ }^{3}$

The reduction of marginal gap to a minimum in crowns and fixed partial dentures is a key goal in prosthodontics. Minimal marginal gaps reduce gingival irritation and cement washout thereby improving clinical outcome and longevity of restoration. ${ }^{4}$ Good marginal fit is one of the most important technical factors for long term success of metal ceramic crowns and this depends on good internal fit. ${ }^{5}$

The purpose of this study is to compare marginal fit of metal copings cast from wax patterns fabricated using conventional wax up technique and CAD/CAM technique.

\section{Materials and Method:-}

Standardized MIS implant abutment (Figure: 1) was taken as die. Sample size of 50 was divided in two groups: 25 wax patterns fabricated by conventional method and 25 wax patterns are fabricated by CAD/CAM.

\section{Fabrication of wax pattern:}

The conventional wax patterns (Kerr Laboratory, CA) fabrication was done by applying two coats of die spacer $1 \mathrm{~mm}$ away from margin with brush system. Dip wax technique (Figure: 2) was used to fabricate the conventional wax copings. After forming a thin layer of wax coping on the die and the inlay wax was added to follow the emergence profile of the die. Finally wax was added at the margins to readapt to the die.

The samples for CAD/CAM (DELCAM) wax patterns were produced with a milling machine unit (ROLAND) using laser scanner and scanner spray (IDS, Germany) which were sprayed on the samples to digitize the dies. Data collected was sent to a software program in which the virtual wax patterns were designed with $0.6 \mathrm{~mm}$ of thickness and $2 \mathrm{~mm}$ of lingual collar height. Simulated die spacer was set at 30 microns starting $1 \mathrm{~mm}$ away from margin. CAM system computed the milling paths and all wax patterns were milled from disc (Roland's DWX-50 disc). The measurement of smallest milling bur for disc was $1 \mathrm{~mm}$.

\section{Investing and casting:}

After attaching wax sprues, all 50 wax patterns were invested in phosphate bonded investment (BEGOSOL) at $24 \mathrm{ml} / 100 \mathrm{~g}$ mixing ratio. The investment was heated upto 950 degrees for eliminating wax and the copings were 
casted in Co-Cr alloy using centrifugal casting machine. The castings were then removed and cleaned with 50 microns aluminium oxide.

\section{Measuring marginal gap:}

The marginal gap was measured under stereomicroscope (MAGNUS) with digital camera attached to personal computer to record measurement. The images were taken in 5 fields around each coping using digital camera. In each field 3 random points were selected so that a total 15 random points will be taken. (Figure: 3 -conventional copings; figure: 4-cad cam copings). Then images were transferred to personal computer for measuring marginal gap at all 15 random points by using image analyzer (IMAGE PRO EXPRESS 6.0).

\section{Results:-}

Mean value of 15 random points were calculated for each coping for both the groups. The mean values are summarized in the below table (Table no: 1) for C1-C25 and M1-M25. The overall mean value for conventional group is $233.415520+/-184.98$ microns and CAD/CAM group 595.256672 +/-264.94 microns. An unpaired t-test was done which revealed highly significant difference between two groups with p value < 0.0001 (Table no: 2 ). Marginal gap for CAD-CAM group was greater at all measured areas when compared to its counter group.

\section{Discussion:-}

The marginal fit of any restoration should be precise and accurate to avoid dissolution of cement and subsequent secondary caries of the abutment teeth. Any marginal gaps in the implant restorations would compromise the seal at the margins and enhance cement dissolution. This leads to constant dislodgement of implant crowns. The purpose of this study was to compare marginal gap of metal copings cast from CAD/CAM wax patterns and conventionally made wax patterns. There was a significant difference in marginal gap between two methods of fabrication. The fit of conventional group is better in all areas than CAD/CAM group.

Physical and mechanical properties of materials have precise definitions whereas the fit of restoration does not have a definition. This lead to considerable variation in defining the reference points for measurements and the descriptive terminology among investigators. Clinical acceptable maximum marginal gap width as established by McLean and Von Fraunhofer is 120 microns. ${ }^{5}$ Moldovan et.al stated 100 microns for marginal misfit as good and 200-300 microns as acceptable. ${ }^{6}$ According to these values, the marginal gap measured in the present study can be rated as good for conventional group than $\mathrm{CAD} / \mathrm{CAM}$ group.

The measurement at the margin is termed as the marginal gap. In the present study the terminology used by Holmes et al. was considered for measuring marginal gap. ${ }^{6}$ Measurements between the castings and the tooth can be made from points along the internal surface at the margin or on the external surface of the casting. There are different techniques followed for measuring marginal gap and internal fit such as direct view technique, Cross sectioning technique, Impression replica technique and Explorer and visual examination. In the present study direct view technique has been used for measuring the marginal gap. This technique measures the gap between crown and die only at the margin and not internally using microscope at different magnifications. This method is considered to be cheaper and less time-consuming than other techniques as it does not incorporate any procedures on the crown-die assembly such as sectioning or replications of the cement space before measuring the gap. The chance of error accumulation that may result from multiple procedures is reduced.

The most important criteria used to evaluate the metal copings is their marginal fit. ${ }^{7}$ Lack of adequate fit causes cement solubility and plaque retention effecting both tooth and supporting periodontal tissues. ${ }^{8}$ As natural teeth show difference in their individual structure, which cause difficulties in attaining standardised abutments, in this study standardized MIS implant abutments were used for measuring marginal gap.

Base metal alloys show good clinical performance and resistance to permanent intraoral deformation. Previously $\mathrm{Co}-\mathrm{Cr}$ alloys were used for RPD frameworks primarily. Currently they are being used for fixed prosthesis, replacing $\mathrm{Ni}-\mathrm{Cr}$ alloys. Evidence from electrochemical studies state that $\mathrm{Co}-\mathrm{Cr}$ alloys are more corrosion resistant than $\mathrm{Ni}-\mathrm{Cr}$ alloys. $\mathrm{Ni}-\mathrm{Cr}$ alloys have a greater sensitization potential than $\mathrm{Co}-\mathrm{Cr}$ alloys, whereas allergies to $\mathrm{Co}-\mathrm{Cr}$ alloys are rare. The absence of allergic response and its rigidity made $\mathrm{Co}-\mathrm{Cr}$ to be selected as material of choice for this study. ${ }^{9}$ 
Milling machine used in this study was 5 milling axis. CAD/CAM systems have introduced some additional steps in fabrication process namely 1.Scanning 2. Software design and 3.Milling and material processing. Beuer et.al reported that quality of restoration does not necessarily increase with number of processing axes. He also stated that factors such as calculation of milling parameters and actual milling process affect fitting accuracy of zirconium restorations, besides scanning procedure of geometric data collected. ${ }^{10}$ The quality of 3D image of tooth preparation is responsible for internal and marginal adaptation of final machined restoration. So, the additional steps of scanning and software limitations could partially explain the larger gap found in CAD/CAM group compared to conventional group. Scanning systems have limitation of finite resolution which can result in edges that are slightly rounded and leads to interfering contacts at incisal/occlusal edges. ${ }^{3}$

Similar results were reported in studies stating the conventional method of fabricating metal copings to be better than restorations fabricated by CAD/CAM, mainly due to marginal fit. Han et al and Tan et al also reported similar results from their study of fabricating titanium crowns where conventional wax up and casting technique produced better marginal fit compared to CAD/CAM technique. ${ }^{12}$ It has also been demonstrated that CAD/CAM system being used has significant effect on marginal fit.

A reverse relation has been observed in previously done research between marginal and internal fit of CAD/CAM systems used. ${ }^{13}$ A large internal gap width have smaller marginal gap dimensions and smaller axial gap could contribute to an under seating restoration and larger marginal gap because of the binding of the restoration and the die. Also most of the cutting tools are incapable of cutting sharp internal angles which results in an internal binding and consequently an open margin.

\section{Limitations of the study}

Firstly, the present study used the method of direct view technique for measuring marginal gap which has few disadvantages like difficulty in selecting the points to measure marginal opening; inability in identifying the apical most part of preparation margin; rounded appearance of the crown margins and die under magnification; less precision due to inaccuracies in repositioning crowns on master die; difficulty in assessing over contouring of crown margin and potential for damage and/or wear of master die on repeated use. Secondly, cementation procedure has not been followed and thirdly, this study measured only the marginal gap whereas internal fit is also needed for more accurate results.

\section{Conclusion:-}

Within limitations of this study and materials and method used following it may be concluded that conventional method of wax pattern fabrication resulted in better marginal fit of final copings than CAD/CAM milled wax patterns. Other findings from the study are that, although CAD/CAM technique has been evolved as the advancement over conventional techniques, much improvement is required in scanning procedure and manufacturing techniques. Additionally, the method of measuring marginal gap may also play major role in obtaining the results.

Table 1:-Mean values for conventional and CAD CAM group

\begin{tabular}{|l|l|}
\hline CONVENTIONAL GROUP MEAN VAUE & CAD/CAM GROUP MEAN VALUE \\
\hline C1-618.1313 & M1-759.5167 \\
C2-977.348 & M2-237.678 \\
C3-135.474 & M3-832.642 \\
C4-124.112 & M4-725.8293 \\
C5-105.8933 & M5-722.094 \\
C6-138.3927 & M6-957.4987 \\
C7-311.228 & M7-915.2853 \\
C8-194.2147 & M8-189.0027 \\
C9-213.7853 $10-145.7787$ & M9- 719.3427 \\
C11-210.00 & M10-686.916 \\
C12-207.984 & M11-712.7527 \\
C13-118.9907 & M12-184.764 \\
C14-113.112 & M13-178.9133 \\
C15- 124.7547 & M14-776.6953 \\
C16-242.101 & M15- 476.68 \\
\hline
\end{tabular}


C17- 157.678

C18-186.1428

C19-195.413

C20-252.794

C21-221.641

C22-189.00

C23-172.996

C24-261.310

C25-217.1128
M17-684.215

M18-910.319

M19-427.7510

M20-195.387

M21-820.616

M22-571.281

M23-493.116

M24-892.471

M25-626.4191

Table 2:-Comparison of the groups

\begin{tabular}{|c|c|c|c|c|}
\hline Group & Mean & SD & SEM & N \\
\hline CAD-CAM Group & 595.256672 & 264.938570 & 52.987714 & 25 \\
\hline CONVENTIONAL Group & 233.415520 & 184.974083 & 36.994817 & 25 \\
\hline
\end{tabular}

Table 3:-Comparison of the groups using unpaired t test

\begin{tabular}{|c|c|c|c|c|}
\hline & $\mathrm{t}$ & $\mathrm{p}-$ value & df & SE \\
\hline Group I vs Group II & 5.5991 & 0.0001 & 48 & 64.624 \\
\hline
\end{tabular}

Figure 1

Figure 2

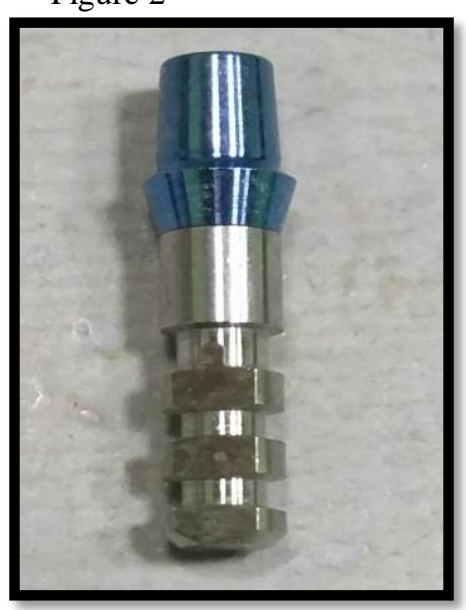

Fig 1:-Standardized MIS implant abutment

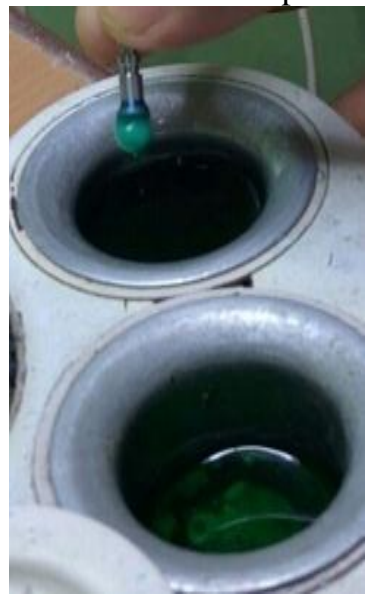

Fig 2:-Wax coping done through dip wax technique 


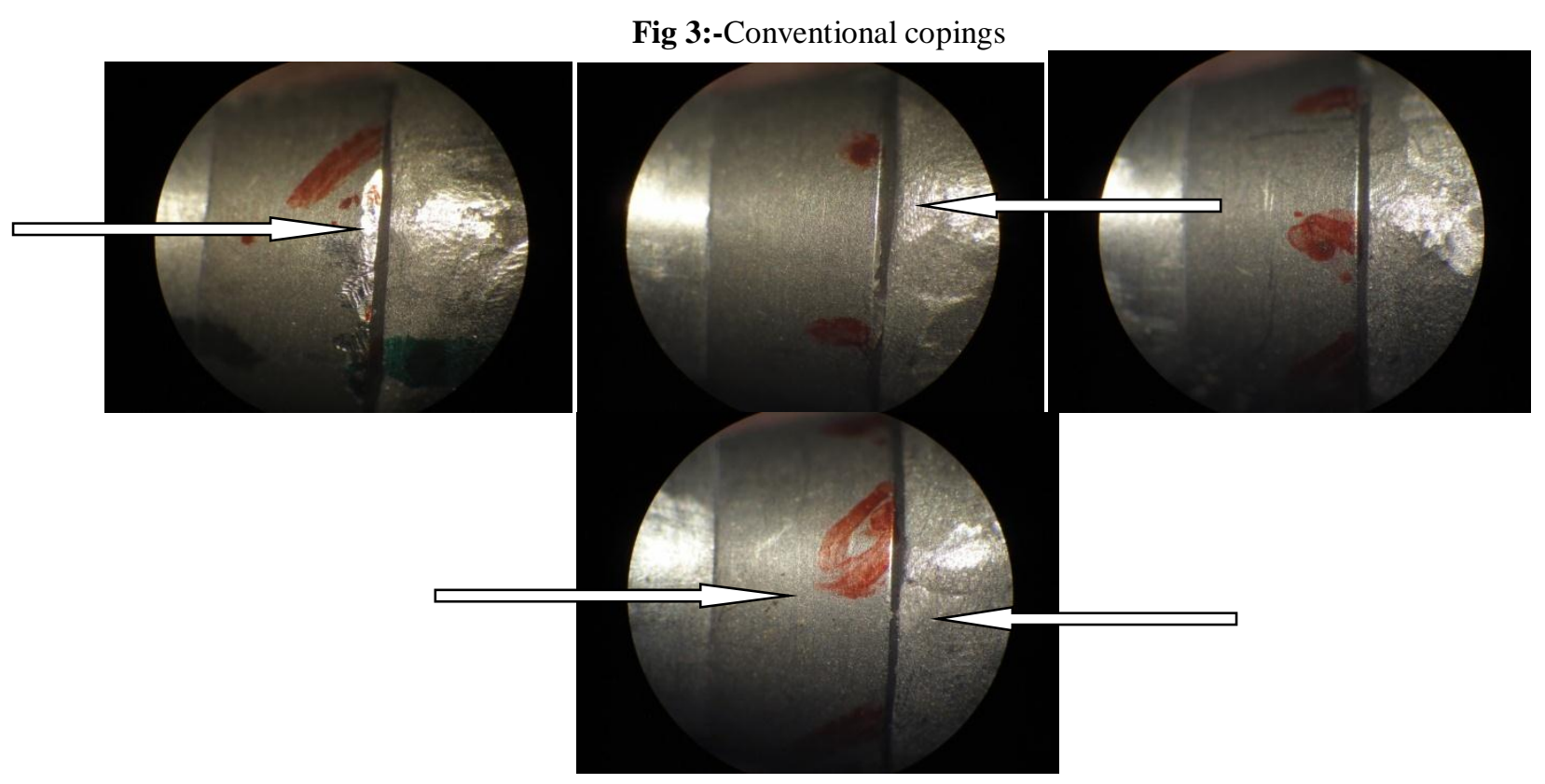

Fig 4:-Cad cam copings
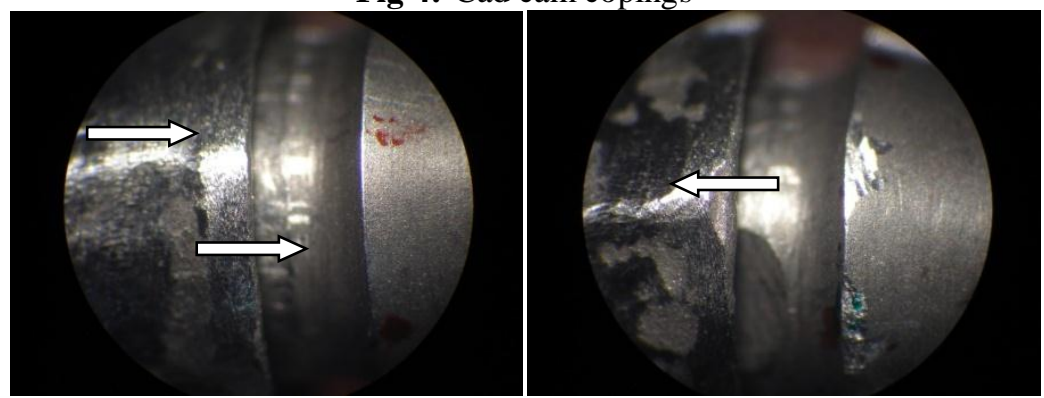

\section{References:-}

1. Vojdani M, Torabi K, Farjood E, Khaledi AA. Comparison the marginal and internal fit of metal copings cast from wax patterns fabricated by CAD/CAM and conventional wax up techniques. Journal of Dentistry. 2013 Sep;14(3):118.

2. Carneiro T. Digital Technology in Implant Dentistry. J Dent \& Oral Disord. 2015; 1(1): 1002.

3. Abduo J, Lyons K, Swain M. Fit of zirconia fixed partial denture: a systematic review. J Oral Rehabil 2010; 37:866-876.

4. Narula S, Punia V, KhaNdelWal M, Sharma V, Pamecha S. Retention in conventional fixed partial dentures: A Review. J Clin Diagn Res. 2011 Oct;5(5):1128-33.

5. Hunter AJ, Hunter AR. Gingival margins for crowns: a review and discussion. Part II: Discrepancies and configurations. J Prosthet Dent 1990; 64: 636-642.

6. Nawafleh NA, Mack F, Evans J, Mackay J, Hatamleh MM. Accuracy and reliability of methods to measure marginal adaptation of crowns and FDPs: a literature review. Journal of Prosthodontics. 2013 Jul;22(5):41928. McLean JW, Von Fraunhofer JA. The estimation of cement film thickness by an in vivo technique. Br Dent J 1971;131:107-111.

7. Holmes JR, Bayne SC, Holland GA, Sulik WD. Considerations in measurement of marginal fit .J Prosthet Dent 1989;62:405-408.

8. Hung SH, Hung KS, Eick JD, and Chappell RP. Marginal fit of porcelain fused to metal and two types of ceramic crown. J Prosthet Dent 1990;63:26-31.

9. Han.HS, Yang.HS, Lim HP, Park.YJ. Marginal accuracy and internal fit of machine milled and cast titanium crowns. J Prosthet Dent 2011;106:191-197. 
10. Stephane V, Francis G.M, Brigitte G: Combination fixed and removable prostheses using a CoCr alloy: A clinical report: J Prosthet Dent 2006; 96:100-3.

11. Tan PL, Gratton DG, Diaz-Arnold AM, Holmes DC. An in vitro comparison of vertical marginal gaps of $\mathrm{CAD} / \mathrm{CAM}$ titanium and conventional cast restorations.

J Prosthodont 2008; 17: 378-383.

12. Beuer F, Schweiger J, Edelhoff D. Digital dentistry: An overview of recent developments for CAD/CAM generated restorations. Br Dent J 2008;204:505-511.

13. Kokubo Y, Nagayama Y, Tsumita M, Ohkubo C, Fukushim S, Vult von Steyern P. Clinical marginal and internal gaps of In-Ceram crowns fabricated using the GN-I system. J Oral Rehabil 2005; 32: 753-758. 